\title{
Quantum Clock Synchronization Based on Shared Prior Entanglement
}

\author{
Richard Jozsa ${ }^{2}$, Daniel S. Abrams ${ }^{1}$, Jonathan P. Dowling ${ }^{1}$, and Colin P. Williams ${ }^{1}$ \\ 1 Jet Propulsion Laboratory, California Institute of Technology, 4800 Oak Grove Drive, Pasadena, California $91109-8099$. \\ 2 Dept. of Computer Science, University of Bristol, Merchant Venturers Building, Woodland Road, Bristol BS8 1 UB, UK.
}

(Received: October 23, 2018)

\begin{abstract}
We demonstrate that two spatially separated parties (Alice and Bob) can utilize shared prior quantum entanglement, and classical communications, to establish a synchronized pair of atomic clocks. In contrast to classical synchronization schemes, the accuracy of our protocol is independent of Alice or Bob's knowledge of their relative locations or of the properties of the intervening medium.
\end{abstract}

PACS: 03.67.-a, 03.67.Hk, 06.30.Ft, 95.55.Sh

In the Special Theory of Relativity, there are two standard methods for synchronizing a pair of spatially separated clocks, $\mathrm{A}$ and $\mathrm{B}$, which are at rest in a common inertial frame. The usual procedure is Einstein Synchronization (ES), which involves an operational line-of-sight exchange of light pulses between two observers, say Alice and Bob, who are co-located with their clocks A and $\mathrm{B}$, respectively [1]. A less commonly used protocol is Eddington's Slow Clock Transport. In this scheme, the two clocks A and B are first synchronized locally, and then they are transported adiabatically (infinitesimally slowly) to their final separate locations [2,3]. A quantum algorithm for efficient clock transport has recently been proposed by Chuang 画.

In this paper we propose a third protocol that utilizes the resource of shared prior entanglement between the two synchronizing parties. Our proposed method of Quantum Clock Synchronization (QCS) has features in common with Ekert's entanglement-based quantum keydistribution protocol [5] in which Alice and Bob initially share only prior-entangled qubit pairs. The key does not exist initially but is created from the ensemble of entangled pairs through a series of measurements and classical messages. Similarly for our QCS protocol below, no actual clocks exist initially but rather only "entangled clocks" in a global state which does not evolve in time. The synchronized clocks are then extracted via measurements and classical communications performed by Alice and Bob. In this way our QCS scheme establishes synchrony without having to transport timing information between Alice and Bob. In contrast, in classical synchronization schemes, actual timing information must be transmitted from Alice to Bob over some channel, whose imperfections generally limit the accuracy of the synchronization.

We begin by reviewing the Ramsey temporal interferometer method [7] for the construction of a quantum clock. A clock is constructed from an ensemble of twolevel systems (qubits) whose temporal evolution proper- ties will determine the time standard. In general any physical qubit may be used. For example, in the International System of Units (SI), the second is defined as the duration of exactly $9,192,631,770$ periods of oscillation corresponding to the hyperfine (radio) transition frequency for the ground-state of the $\mathrm{Cs}^{133}$ atom [6]. Below we also consider other possible physical realisations of the qubit.

Consider a qubit with stationary states $|0\rangle$ and $|1\rangle$ having energy eigenvalues $E_{0}<E_{1}$ respectively. We introduce the dual basis $\mid$ pos $\rangle=\frac{1}{\sqrt{2}}(|0\rangle+|1\rangle)$ and $\mid$ neg $\rangle=$ $\frac{1}{\sqrt{2}}(|0\rangle-|1\rangle)$ and write $\Omega=\frac{1}{\hbar}\left(E_{1}-E_{0}\right)$. The Hadamard transform $H$ is defined by the operation $|0\rangle \rightarrow \mid$ pos $\rangle$ and $|1\rangle \rightarrow \mid$ neg $\rangle$. Let us write $\sigma_{3}$ for the measurement in the $\{|0\rangle,|1\rangle\}$ basis and $\sigma_{1}$ for the measurement in the dual basis. Thus if the qubit is a spin $\frac{1}{2}$ particle in a $z$-oriented magnetic field then $|0\rangle$ and $|1\rangle$ are the $z$ spin eigenstates with $\sigma_{3}$ and $\sigma_{1}$ being $\sigma_{z}$ and $\sigma_{x}$ respectively. If the qubit comprises two hyperfine energy levels of a $\mathrm{Cs}^{133}$ atom, then $\sigma_{3}$ measures population in these levels and $\sigma_{1}$ is measured by first applying $H$ and then measuring $\sigma_{3}$.

The Ramsey method for providing a time standard is based simply on the fact that the states $|p o s\rangle$ and $|n e g\rangle$ are not stationary states. They evolve in time as:

$$
\begin{aligned}
& |\operatorname{pos}(t)\rangle=\frac{1}{\sqrt{2}}\left(e^{-i \Omega t / 2}|0\rangle+e^{i \Omega t / 2}|1\rangle\right) \\
& |n e g(t)\rangle=\frac{1}{\sqrt{2}}\left(e^{-i \Omega t / 2}|0\rangle-e^{i \Omega t / 2}|1\rangle\right)
\end{aligned}
$$

At some time $t=0$ we apply $H$ to an ensemble of qubits in state $|0\rangle$ giving an ensemble of states $|p o s\rangle$ which begin to evolve as in eq. (1). After a time $t$ we measure the observable $\sigma_{1}$ (either directly or by first applying $H$ and measuring $\sigma_{3}$, depending on the physical implementation of the qubits). A straightforward calculation shows that the probabilities for seeing outcomes 0 or 1 are given by

$$
P(0)=\frac{1}{2}(1+\cos (\Omega t)), \quad P(1)=\frac{1}{2}(1-\cos (\Omega t))
$$

By monitoring the oscillations of either $P(0)$ or $P(1)$ as a function of time we get an estimate of the clock phase $\Omega t \bmod 2 \pi$ and hence of $t$.

We now describe our proposed QCS scheme. We assume at the outset that Alice and Bob share an ensemble of singlet states $\left|\psi^{-}\right\rangle=\frac{1}{\sqrt{2}}\left(|0\rangle_{A}|1\rangle_{B}-|1\rangle_{A}|0\rangle_{B}\right)$ where the subscripts refer to particles held by Alice and Bob. The pairs are labelled $n=1,2,3, \ldots$ and the labels are known to both Alice and Bob. This singlet state is a "dark state" that does not evolve in time provided A and 
B undergo identical unitary evolutions. Indeed for any 1-qubit unitary $U$ we have $(U \otimes U)\left|\psi^{-}\right\rangle=(\operatorname{det} U)\left|\psi^{-}\right\rangle$ so that $\left|\psi^{-}\right\rangle$changes only by an overall unobservable phase. Our protocol below (slightly modified) would work equally well using the state

$$
\left|\psi^{-}(\eta)\right\rangle=\frac{1}{\sqrt{2}}\left(|0\rangle_{A}|1\rangle_{B}-e^{i \eta}|1\rangle_{A}|0\rangle_{B}\right)
$$

for any fixed $\eta$. This state still has the essential property of being constant in time i.e. invariant under $U \otimes U$ where $U$ is time evolution, diagonal in the $\{|0\rangle,|1\rangle\}$ basis (but unlike the singlet, it is not invariant under $U \otimes U$ for more general $U$ 's).

We will refer to a pair of clocks in the singlet state $\left|\psi^{-}\right\rangle$ as an entangled pair of pre-clocks. Since $\left|\psi^{-}\right\rangle$is constant in time the pre-clock pairs could be said to be "idling" they can provide no direct timing information. We may also write $\left|\psi^{-}\right\rangle$in the $\sigma_{1}$ measurement basis as

$$
\left.\left|\psi^{-}\right\rangle=\frac{1}{\sqrt{2}}(\mid \text { pos }\rangle_{A}|n e g\rangle_{B}-|n e g\rangle_{A}|p o s\rangle_{B}\right)
$$

Let $t$ be a time coordinate in the common rest frame of Alice and Bob. To start the clocks at some time $t=0$, Alice simultaneously measures all of her pre-clock pairs in the $\sigma_{1}$ basis $\{|p o s\rangle,|n e g\rangle\}$. Thus each pair collapses randomly and simultaneously at $A$ and $B$ into one of the following states:

$$
\begin{aligned}
& \left.\left.\left|\psi^{I}\right\rangle=\mid \text { pos }\right\rangle_{A} \mid \text { neg }\right\rangle_{B} \\
& \left.\left.\left|\psi^{I I}\right\rangle=\mid \text { neg }\right\rangle_{A} \mid \text { pos }\right\rangle_{B}
\end{aligned}
$$

with equal probability $\frac{1}{2}$. The $A$ and $B$ clocks begin to evolve in time, in accordance with Eq. (ID) - all starting synchronously at a time $t=0$ in Alice and Bob's shared inertial frame. Indeed Alice's measurement effectively reproduces the result of the first one-clock Hadamard transform in the Ramsey scheme. However the result here is a mixture of two equally weighted sub-ensembles I and II. As a result of her measurement, Alice knows the labels belonging to the subensembles I and II but Bob is unable to distinguish them.

The density matrix of Bob's overall ensemble is still $\rho=\frac{1}{2} I$, independent of $t$, so no measurement statistic can provide Bob with any timing information. For Bob to extract a clock, a classical message from Alice is required. Alice post-selects from her entire ensemble the sub-ensemble of Type-I qubits. Since the qubits are labelled, she can then tell Bob which subset of his qubits are also Type-I by broadcasting their labels via any form of classical communiqué. Bob is then able to extract his own Type-I and Type-II subensembles. Choosing the Type-II subensemble, Bob will have a clock ensemble exactly in phase with a Type-I clock that Alice started at $t=0$. To establish synchrony, Bob measures $\sigma_{1}$ on this ensemble (either directly or by applying $H$ and measuring $\left.\sigma_{3}\right)$ and monitors the oscillations of $P(0)$ as in eq.
(2). Alice and Bob now have clocks that are ticking in unison.

The protocol as described above is still incomplete [9] because of the following rather subtle point: there are extra hidden assumptions in the requirement that Alice and Bob are both able to perform the same $H$ operation and identify the same $|p o s\rangle$ states. Indeed if we are given only $|0\rangle$ and $|1\rangle$ as physical states (i.e. normalised vectors up to overall phase) then the physical states $|p o s\rangle$ and $|n e g\rangle$ are not uniquely determined円 and so $H$ is also not uniquely determined (as, for example, it entails knowledge of $|p o s\rangle)$. A further arbitrary choice needs to be made, analogous to a choice of reference frame, to fix these further constructs.

The need for a further choice is most clearly seen by considering the spin $\frac{1}{2}$ qubit [8]. The physical states $|0\rangle$ and $|1\rangle$ define a $z$ direction and $|p o s\rangle$ defines a perpendicular $x$ direction. But given only a $z$ direction we are free to choose any orthogonal direction as $x$. On the Bloch sphere $|0\rangle$ and $|1\rangle$ are two given poles and $\mid$ pos $\rangle$ may be arbitrarily chosen to be any point on the equator. Once $|p o s\rangle$ is chosen, it must be consistently used in all future operations. Furthermore, there is then no further ambiguity in the identity of any state on the Bloch sphere e.g. $|n e g\rangle$ and $H$ are then uniquely fixed.

The same remarks apply to the Cs atom qubit but the physical interpretation is quite different. The operation $H$ (and hence $|p o s\rangle$ ) is physically defined in terms of a $\pi / 2$ pulse applied to the physical state $|0\rangle$. But this pulse has an origin of phase which must be chosen and then fixed ("locked") for all future applications of $H$. Different choices of phase locking correspond to different choices of points on the Bloch sphere equator for $|p o s\rangle$. Note that a choice of phase locking here corresponds physically to a choice of time origin in contrast to the spin $\frac{1}{2}$ qubit, where the choice was a spatial direction.

For our QCS protocol to work correctly, Alice and Bob must use the same choice of physical state $|p o s\rangle$ (or equivalently use the same choice of Hadamard operation $H$ ). If they use two different choices (and use them locally consistently) then their clocks will not be ticking in synchrony, but be offset by an amount depending on the angle between the two choices of $|p o s\rangle$ on the Bloch equator.

In the physical implementation given by the Cs atom qubit, a consistent choice of $H$ requires that Alice and Bob have mutually phase locked pulses. But this is equivalent to them having clocks ticking in synchrony thus defeating the purpose of the protocol! However the follow-

\footnotetext{
${ }^{1}$ Note that if $|0\rangle$ and $|1\rangle$ are given as vectors then all other vectors such as $|p o s\rangle$ are uniquely defined so our ambiguity depends essentially on the fact that a physical state is not just a (normalised) vector but rather, a set of all such vectors that differ by an overall phase.
} 
ing extension of our protocol gets around this difficulty, allowing Alice and Bob to establish time synchrony without the resource of mutually phase locked pulses: we duplicate our protocol above for two different values $\Omega_{1}$ and $\Omega_{2}$ of $\Omega$ e.g. we use two different species of atoms. Thus Alice and Bob will require two different kinds of pulses for the two frequencies. In his laboratory, Bob is able to lock the phases of his two pulses, and similarly for Alice, so there will be a common offset $\delta$ between the two locked settings of Alice and Bob. By measuring populations in state $|0\rangle$ as before, Alice will have the oscillations:

$$
P_{1}^{A}=\frac{1}{2}\left(1+\cos \Omega_{1} t\right) \quad P_{2}^{A}=\frac{1}{2}\left(1+\cos \Omega_{2} t\right)
$$

and Bob's oscillations will be offset by the constant (unknown) $\delta$ :

$$
P_{1}^{B}=\frac{1}{2}\left(1+\cos \left(\Omega_{1} t+\delta\right)\right) \quad P_{2}^{B}=\frac{1}{2}\left(1+\cos \left(\Omega_{2} t+\delta\right)\right)
$$

But now by observing the beats between the two oscillations $P_{1}$ and $P_{2}$ Alice and Bob are able to establish synchronously ticking clocks. Indeed we have

$$
P_{1}^{B}-P_{2}^{B}=\sin \left(\frac{1}{2}\left(\Omega_{1}-\Omega_{2}\right) t\right) \sin \left(\frac{1}{2}\left(\Omega_{1}+\Omega_{2}\right) t+\delta\right)
$$

so that the envelope (given by the first term) oscillates independently of $\delta$ exactly in phase with Alice's corresponding envelope.

It is interesting to consider the above problem, of locally consistent but different choices of $|p o s\rangle_{A}$ and $|p o s\rangle_{B}$, in the alternative physical scenario of clocks given by ensembles of spin $\frac{1}{2}$ qubits in a magnetic field. Although mathematically equivalent, we will see that the physical implications are quite different. In this scenario $|0\rangle$ and $|1\rangle$ are the $z$ spin eigenstates. We imagine that a third party (Clare) prepares an ensemble of pairs in the singlet state and simultaneously puts each spin in a labelled box containing a constant magnetic field $B_{z}$ in the $z$ direction. She then distributes the boxes (complete with their magnetic fields) to Alice and Bob (appropriately for each pair). Note that Alice and Bob may determine the $z$ direction (if they do not already know it) by measuring the direction of the (classical) magnetic field in a box (without disturbing the particle).

Alice now chooses an $x$ direction (perpendicular to $z$ ) and at some time $t=0$ she measures $\sigma_{x}$ on all her particles. Then, just as before, Bob may establish synchrony by monitoring the oscillations of $\sigma_{x}$ measurement outcomes on a sub-ensemble of his particles (selected by classical information from Alice). The previous problem of consistent phase locked pulses becomes the problem of Bob choosing the same $x$ direction that Alice used. Previously, the problem was equivalent to the original goal of the protocol (time synchrony) but here it is different i.e. a requirement of space parallelism ("space synchrony"). This allows the possibility of new physical resolutions of the problem, not available for Cs atom qubits. For example, Alice and Bob may have a prior agreement to use the direction to the pole star as their $x$ direction (which would be parallel to high accuracy for any two locations on Earth) i.e. $x$ - "space synchrony" may be given for free, whereas time synchrony is not.

The idea of the previous resolution - using two frequencies - may be used for spin $\frac{1}{2}$ qubits as well (e.g. if Alice and Bob are unable to see any fixed stars.) Clare sets up boxes with two different magnetic fields (both in the $z$ direction) giving the two different frequencies. Bob chooses his $x$ axis randomly (perpendicular to $z$ ) and the constant phase offset $\delta$ now arises from the fixed angle between Alice's and Bob's chosen $x$ directions. An important point here is that different physical realisations of a qubit - although mathematically equivalent - lead to quite different avenues for getting around limitations of a (mathematically) given protocol.

For some applications, such as satellite-based Very Long Baseline Interferometry (VLBI) [10], the fact that Alice and Bob's clocks are phase locked up to only modulo $2 \pi$ is sufficient. However, there are other applications, such as the synchronization of satellite-borne atomic clocks in the Global Positioning System (GPS) [11], where it is important to have a shared origin of time. For such applications, we may adapt our QCS protocol to construct a common temporal point of reference as follows. Using the protocol Alice and Bob set up clocks ticking synchronously for two different frequencies $\Omega_{1}$ and $\Omega_{1}+\Delta \Omega$. The envelope of beats between these frequencies oscillates with frequency $\frac{1}{2} \Delta \Omega$. If the protocol for establishing the two ticking synchronisations is completed in time $T$ and $\Delta \Omega$ is chosen so that $\Delta \Omega T<\frac{\pi}{2}$ then Alice and Bob may determine a unique common time origin as the first maximum of the beat oscillations.

There are several immediate applications and advantages of our QCS protocol. For example, in the GPS satellite constellation, the ability of the space-borne atomic clocks to synchronize with a master atomic clock on the ground is affected by the fluctuating refractive index of the atmosphere, causing random variations in the speed of light and limiting the accuracy of the classical ES protocol. This index fluctuation error is the current limiting factor of GPS precision [11]. With our QCS scheme, the properties of the atmosphere have no effect. In fact, Alice and Bob need not even have exact knowledge of their relative locations.

Also classical ES requires the exchange and timing of light pulses, but light is actually a quantum field. Hence the arrival time of a light pulse is itself subject to quantum fluctuations, limiting the accuracy of the ES protocol [13]. In contrast, our QCS scheme is unaffected by this kind of noise.

The Ramsey two-pulse temporal interferometer is iso- 
morphic, via the $\mathrm{SU}(2)$ algebra, to an optical or matterwave Mach-Zehnder interferometer 14. Hence, the QCS protocol may be readily adapted to the task of phase locking a pair of spatially separated optical or atom interferometers, with applications to various forms of interferometry such as VLBI.

A shortcoming of our QCS protocol that it does not specify a method by which the shared prior entanglement between Alice and Bob may be established. One possibility is for Alice and Bob to meet at a common location, create an ensemble of $N$ identical EPR pairs each in the state $\frac{1}{\sqrt{2}}\left(|0\rangle_{A}|1\rangle_{B}-|1\rangle_{A}|0\rangle_{B}\right)$ and then go their separate ways. But then, could not Alice and Bob just establish time synchrony at their meeting and retain accurate clocks for future use, instead of carrying the entanglement? In practice, clocks drift and periodic corrections of synchronisation will be necessary, suffering from the limitations of the classical schemes. It is not clear whether the task of carrying and maintaining the required entanglement is equivalent to the task of carrying and maintaining accurately running clocks. One difference is that in the former case, the time synchrony does not initially exist but is set up only when required, which may have applications for security.

An alternative scheme for establishing the shared prior entanglement would not require Alice and Bob to meet at all. Instead, it would involve Alice and Bob each receiving corresponding members of EPR pairs from some common source and then using entanglement purification [15] to distill them into an ensemble of singlet states as required by QCS. Unfortunately, there is a hidden assumption of simultaneity in the actions to be performed by Alice and Bob in the current entanglement purification protocols when the states $|0\rangle$ and $|1\rangle$ are nondegenerate in energy 16, as required in our protocol. This means ultimately that the existing entanglement purification schemes can only create states of the form $\left|\psi^{-}(\eta)\right\rangle$, where $\eta$ is unknown, rather than the true singlets (or states with known $\eta$ ) needed for QCS. We are currently investigating whether we can use such states in a modified version of QCS or indeed whether there are alternative (asynchronous) entanglement purification protocols that can produce pure singlets.

A second limitation of our protocol is the requirement that Alice and Bob be relatively at rest. In a more realistic scenario we would need to assess and correct the effects of relative motions and accelerations, especially on the exact form of the entanglement existing between Alice and Bob.

In conclusion, we have presented a quantum protocol for synchronizing spatially separated atomic-clocks, which uses only shared prior entanglement and a classical channel. The two synchronizing parties may be at far-distant and unknown relative locations and the accuracy of the time synchronisation is not affected by the distance of separation or by noise on the classical channel. Our protocol has direct applications for use in very long baseline interferometry and also provides a means for phase locking remote optical or matter-wave interferometers.

\section{ACKNOWLEDGMENTS}

We would like to acknowledge valuable discussions with S. L. Braunstein, N. Cerf, J. I. Cirac, H. J. Kimble, N. Linden, H. Mabuchi, L. Maleki, S. Popescu, J. P. Preskill, and N. Yu.

D.S.A., J.P.D. and C.P.W. are supported by a contract with the National Aeronautics and Space Administration. R. J. is supported by the U.K. Engineering and Physical Sciences Research Council.

[1] A. Einstein, Ann. D. Physik 17, 891 (1905). Einstein. The Swiss Years: Writings, 1900-1909, Vol. 2 (Princeton University Press, Princeton, NJ, 1989), pp. 140-171.

[2] A.S. Eddington, The Mathematical Theory of Relativity, 2nd ed., Cambridge University Press, , 1924.

[3] R. Anderson, I. Vetharaniam, and G.E. Stedman, Phys. Rep. 295, 93 (1998).

[4] I. Chuang, preprint available at http://xxx.lanl.gov/quant-ph/0005092

[5] A.K. Ekert, Phys. Rev. Lett. 67, 661 (1991).

[6] C. Audoin, Metrologia 29, 113 (1992).

[7] N.F. Ramsey, Molecular Beams, Oxford University Press, Oxford, U.K., (1969), Sec. V.4.

[8] We are grateful to S. Popescu for clarifying this point.

[9] We are grateful to J. I. Cirac for drawing our attention to this problem.

[10] G.S. Levy, et al., Acta Astronaut. 15, 481 (1987).

[11] National Research Council Staff, The Global Positioning System: A Shared National Asset, National Academy Press, Washington, DC, (1995).

[12] I.I. Sobelman, Atomic Spectra and Radiative Transitions, 2nd ed. Springer-Verlag, Berlin, (1992), Sec. 6.2.5.

[13] M.T. Jaekel and S. Reynaud, Phys. Rev. Lett. 76, 2407 (1996).

[14] J.P. Dowling, Phys. Rev. A 57, 4736 (1998).

[15] C. H. Bennett, G. Brassard, S. Popescu, B. Schumacher, J. Smolin and W. Wootters, Phys. Rev. Lett. 76, 722 (1996).

[16] J.P. Preskill private communication. 